\title{
Webbed Penis Associated with Urethral Duplication: A Case Report
}

\author{
Burhan Aksu, Mustafa İnan, Mehmet Pul \\ Department of Pediatric Surgery, Faculty of Medicine, Trakya University, Edirne, Turkey
}

\begin{abstract}
Urethral duplication and webbed penis are rare congenital anomalies. Urethral duplication associated with webbed penis has not previously been reported in the literature. We describe a case of incomplete urethral duplication with webbed penis in an infant and discuss the clinical and radiological findings and treatment of this association.
\end{abstract}

Key Words: Webbed penis, urethral duplication, urethroplasty

Received: 24.11.2008 Accepted: 06.02.2009

Fusion of the penile and scrotal skin, known as webbed penis, is a rare and little-known defect of the external genitalia. Accessory urethra, or duplication of the urethra, is an extremely rare anomaly. We report a case of webbed penis associated with urethral duplication in an infant and discuss its clinical features and surgical treatment.

\section{Case Report}

An 18-month-old boy was admitted to our hospital due to the abnormal appearance of the external genitalia. The boy was born at full term after an uneventful pregnancy, and weighed $3400 \mathrm{~g}$ with a length of $51 \mathrm{~cm}$ and a head circumference of $35 \mathrm{~cm}$. The patient's history showed that his mother first noticed the abnormal external genitalia at birth, and this was confirmed by his father. After a period of time, his general practitioner referred him to our hospital for surgical treatment. The mother's pregnancy was uncomplicated and there was no exposure to drugs, alcohol, or other known toxins.

Physical examination showed a web of skin between the scrotum and the urethral surface of the penis, and proximal incomplete urethral duplication (Figs. 1A, B). There was no chordee. The excretory urography showed Type I incomplete urethral duplication (Fig. 2A). The results of micturating cystourethrography (MCU) (Fig. 2B) and other laboratory tests were normal. In the operation, the web was incised transversely and sutured vertically. Urethroplasty was performed to repair the incomplete urethral duplication. Circumcision was also performed. He had no problems in the 15 months following the operation.

\section{Discussion}

Normal embryological development of the male urethra is completed by the third month. The genital tubercle elon- gates, and on its ventral aspect, a urethral plate of ectodermal derivation is covered by lateral genital folds to complete the urethral tube. The glans penis develops by an invagination of ectoderm, which communicates with the urethra proximally.

The anatomy of malformations in urethral duplication varies so widely that a single hypothesis is unlikely to offer a satisfactory explanation for all types. Psihramis (1) proposes a theory that explains the majority of cases. A partial failure or irregularity in the ingrowth of the lateral mesoderm between the ectoderm and endoderm of the cloacal membrane can account for the dorsal double channel. The distal dorsal duplication can be explained by canalisation of the urethral plate and perineal duplication can be due to defective uro-rectal fold development. Accessory urethral opening into an accessory meatus could be due to an ectodermal ingrowth for the glans. The embryology of webbed penis is unknown; however, a disturbance in the normal preputial development described by Hunter (2) may explain the deficiency of ventral penile skin.

The anatomical variations of a duplicated urethra have been classified and modified by various authors $(3,4)$. Effermann et al (4) have offered the most complete classification:

Type I. Incomplete urethral duplication

Type II. Complete urethral duplication

a. Two meatuses

(i) Non-communicating urethras arising independently from the bladder

(ii) A second channel arising from the first and exiting independently

b. Complete duplication joining at one meatus

Type III. Complete duplication as a component of caudal duplication

Our patient belongs to Type I of this classification system.

Congenital abnormalities associated with urethral duplication are present in most cases. Duplication of the bladder, uterus, vagina, bifid clitoris, scrotum, terminal ileum, colon, 


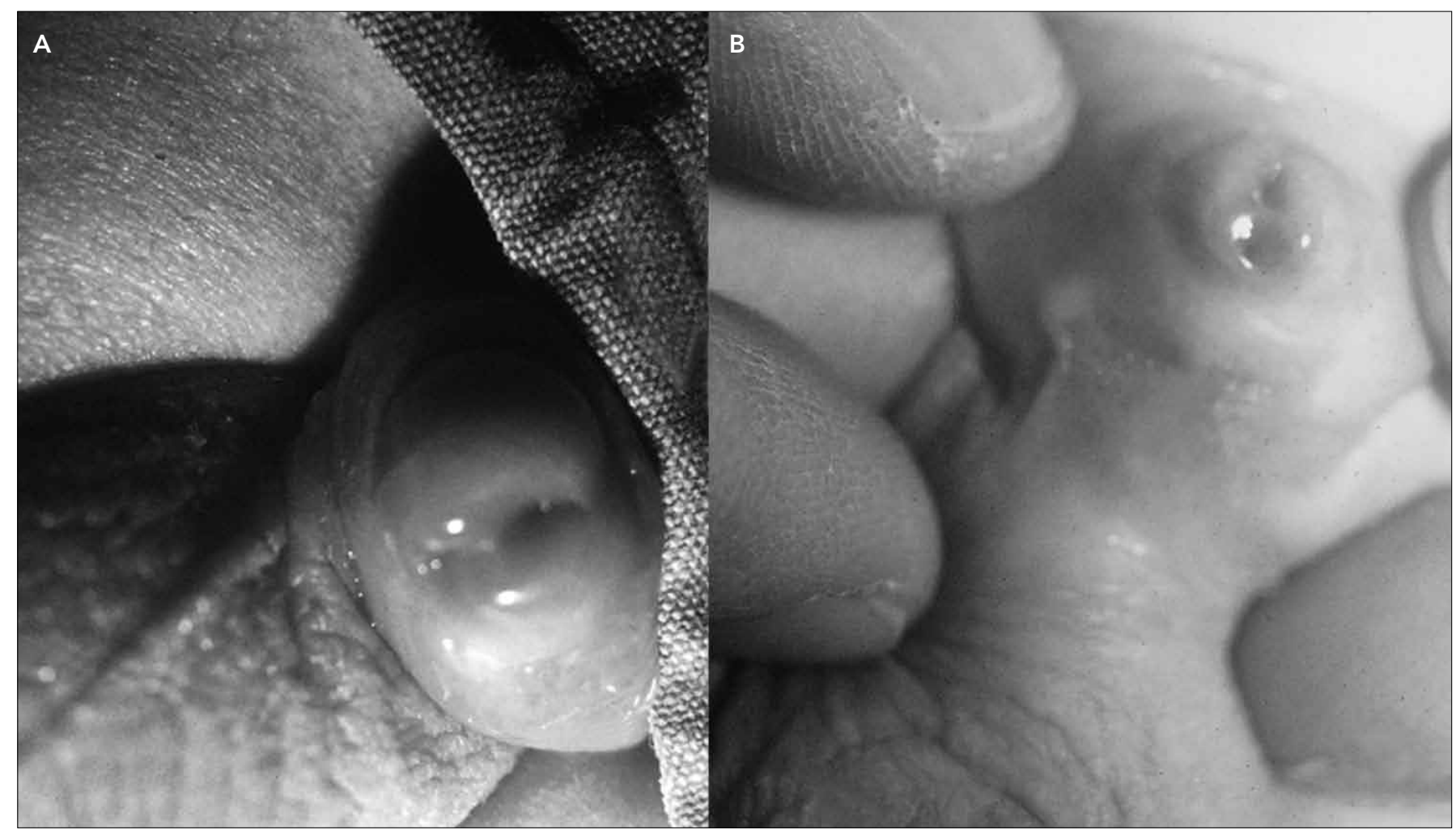

Figure 1. (A) Appearance of urethral duplication, and (B) the penoscrotal fusion (webbed penis) is apparent

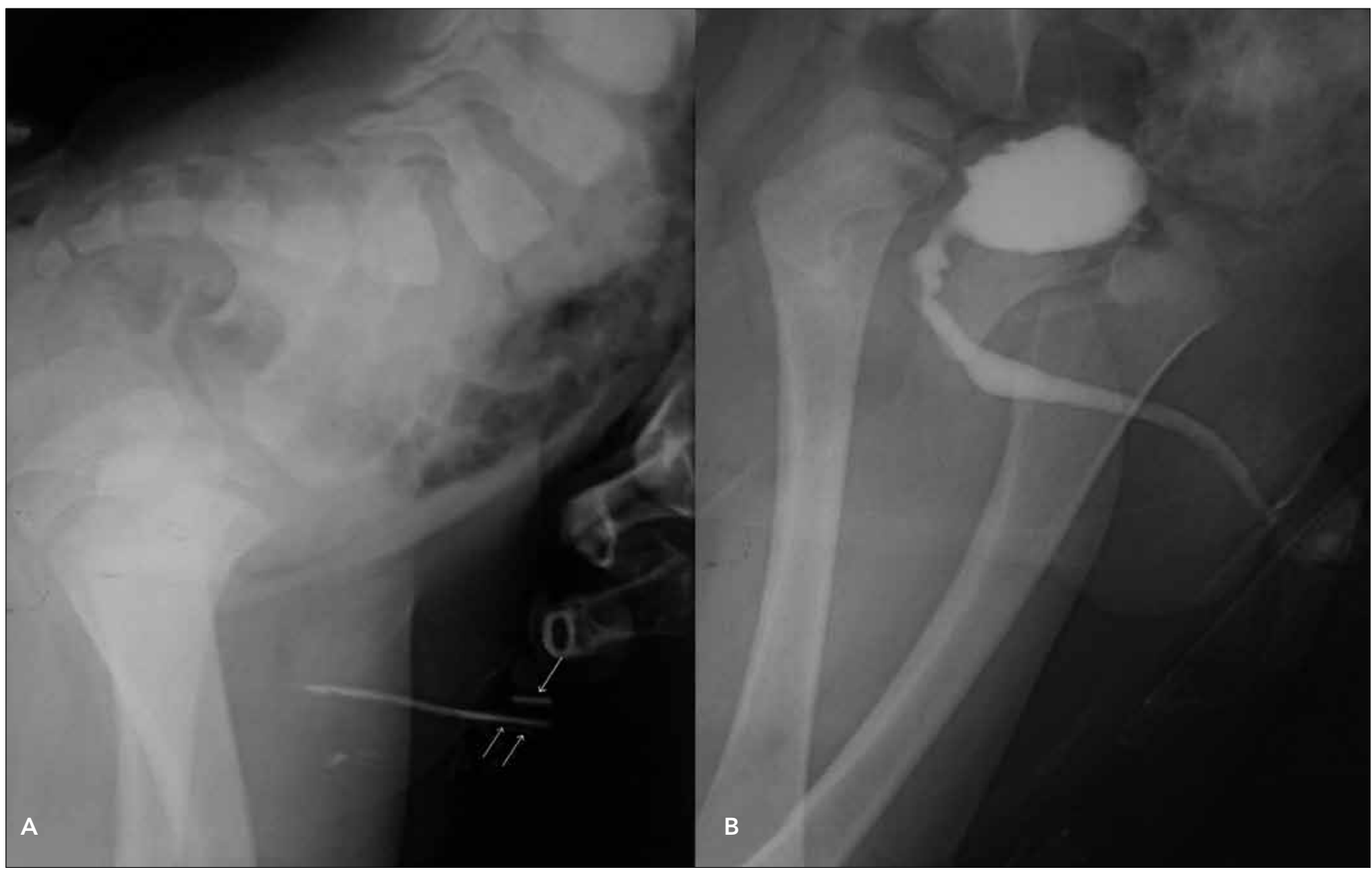

Figure 2. (A) The excretory urography of the infant shows Type I incomplete urethral duplication [ $\uparrow$ Type I incomplete urethral duplication; $\uparrow \uparrow$ Normal urethral canal] (B) Normal MCU 
and rectum, as well as anal atresia, anterior ectopic anus, mental retardation, spinal anomalies, diplopodia of the foot, intestinal malrotation, and umbilical hernia have been reported in the literature (5-8). We could not find any case of webbed penis associated with urethral duplication.

Webbed penis is diagnosed by inspection, but the diagnosis of urethral duplication is more difficult. It can be made by clinical examination, urethrography, $\mathrm{MCU}$, and types of excretory urography. These diagnostic procedures are also essential for identification and confirmation of associated anomalies (9). Urethral duplication is more common in children than adults and most patients are asymptomatic, as in our case. However, symptoms depend on the type of duplication and vary from a double stream, infections, and incontinence to, rarely, straining due to extrinsic compression of the potent urethra. Webbed penis involves sexual difficulties and voiding problems.

We think that urethroplasty is a perfect surgical procedure for treatment in Type I urethral duplication cases. At the same time, webbed penis can be treated easily with transverse incision and vertical closing of the penoscrotal fusion line, as in our case. Our patient's post-operative cosmetic appearance was excellent and he has not had any voiding problems.

\section{Conflict of Interest}

No conflict of interest was declared by the authors.

\section{References}

1. Psihramis KE, Colodny AH, Lebowitz RL, Retik AB, Bauer SB. Complete patent duplication of the urethra. J Urol 1986;136:63-7.

2. Hunter RH. Notes on the development of the prepuce. J Anat 1935;70:68.

3. Das S, Brosman SA. Duplication of the male urethra. J Urol 1977;117:452-4.

4. Effmann EL, Lebowitz RL, Colodny AH. Duplication of the urethra. Radiology 1976;119:179-85.

5. Bellagha I, Chaouachi B, Hammou A, Dhaoui R, Kaabar N, Aloulou $T$ et al. An exceptional combined malformation: duplication of the lower urinary tract, the vulva and the posterior intestine Ann Urol Paris 1993;27:101-5.

6. Goh DW, Davey RB, Dewan PA. Bladder, urethral, and vaginal duplication. J Pediatr Surg 1995;30:125-6.

7. Singal AK, Bhatnagar V, Agarwala S, Mitra DK. Urethral duplication in association with anorectal malformation. Eur J Pediatr Surg 2006;16:214-6.

8. Voigt HR, Wentzel SW. Complete duplication of the bladder, urethra and external genitalia in a male neonate with an imperforate anus. Int J Urol 2005;12:702-4.

9. Mutlu N, Baykal M, Merder E, Culha M, Canbazoglu N. Diphallus with urethral duplication: a rare case. Int J Clin Pract 1997;5:525-6. 